\title{
Finite Difference Micromagnetic Simulation with Self-Consistent Currents and Smooth Surfaces
}

\author{
Mathew R. Gibbons \\ Greg Parker \\ Charles Cerjan \\ Dennis W. Hewett
}

This paper was prepared for submittal to the

2nd International Symposium on Hysteresis Modeling \& Micromagnetics

Perugia, Italy

June 7-9, 1999

May 27, 1999

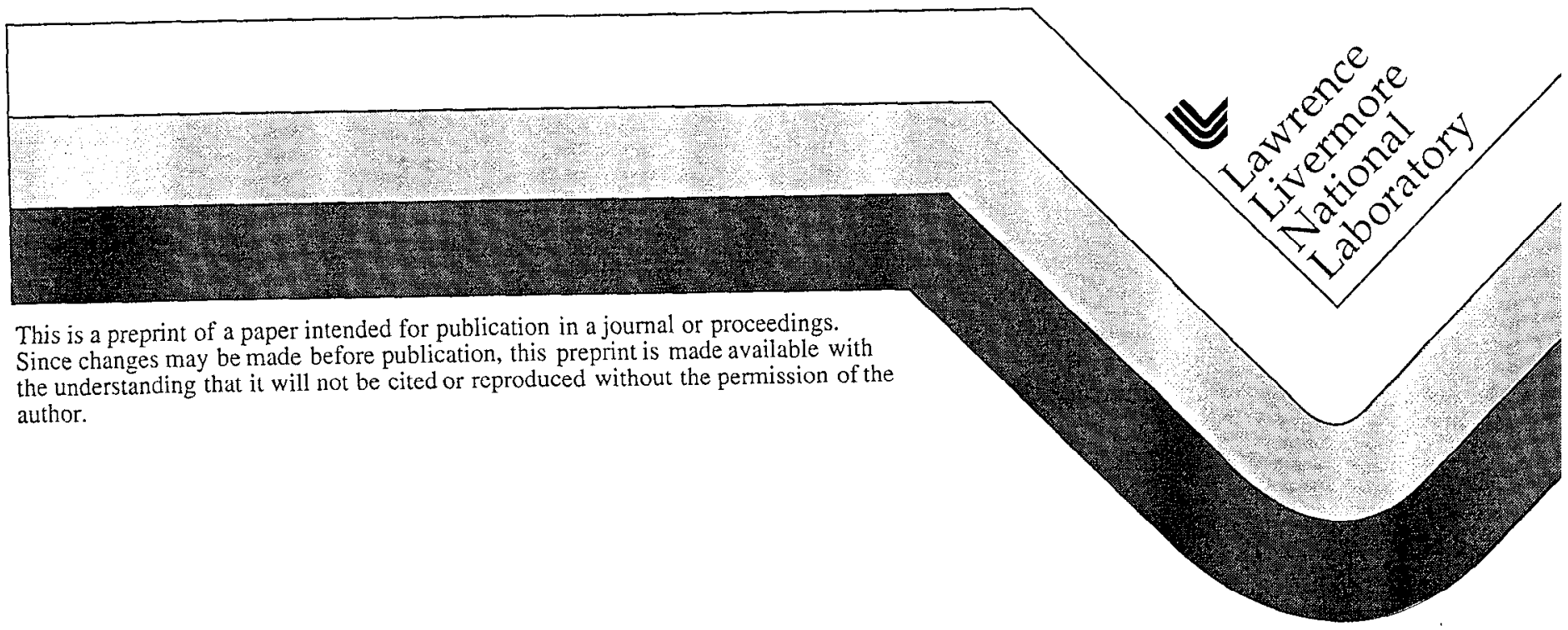




\section{DISCLAIMER}

This document was prepared as an account of work sponsored by an agency of the United States Government. Neither the United States Government nor the University of California nor any of their employees, makes any warranty, express or implied, or assumes any legal liability or responsibility for the accuracy, completeness, or usefulness of any information, apparatus, product, or process disclosed, or represents that its use would not infringe privately owned rights. Reference herein to any specific commercial product, process, or service by trade name, trademark, manufacturer, or otherwise, does not necessarily constitute or imply its endorsement, recommendation, or favoring by the United States Government or the University of California. The views and opinions of authors expressed herein do not necessarily state or reflect those of the United States Government or the University of California, and shall not be used for advertising or product endorsement purposes. 


\title{
Finite Difference Micromagnetic Simulation with Self-Consistent Currents and Smooth Surfaces
}

\author{
Matthew R. Gibbons ${ }^{a}$, Greg Parker ${ }^{b}$, Charles Cerjan ${ }^{b}$, Dennis W. Hewett ${ }^{b}$ \\ ${ }^{a}$ Read-Rite Corporation, 44100 Osgood Road, Fremont, CA 94539, USA \\ ${ }^{\mathrm{b}}$ Lawrence Livermore National Laboratory, Livermore, CA 94550, USA
}

\begin{abstract}
A micromagnetic algorithm has been developed using the finite difference method (FDM). Elliptic field equations are solved on the mesh using the efficient Dynamic Alternating Direction Implicit method. Smooth surfaces have been included in the FDM formulation so structures of irregular shape can be modeled. The current distribution and temperature of devices are also calculated.

Keywords: Micromagnetic simulation, Magnetic dots, Read heads, Thermal Effects

Corresponding author:

Matthew R. Gibbons, Read-Rite Corporation, 44100 Osgood Road, Fremont, CA 94539, USA

phone: (510) 683-7732,fax: (510) 683-7065, e-mail: matt.gibhons@readrite.com

\section{INTRODUCTION}

This paper describes the enhancement of a micromagnetics algorithm to include smooth surfaces, selfconsistent currents, and temperature. Section II provides a summation of the additional techniques that have been implemented. A more detailed description of the initial code can be found in [1]. Results for the smooth surfaces and self-consistent currents are investigated in the remainder of the paper. Section III presents the comparison of simulation with experimental results for micron and submicron size ferromagnetic particles. In section IV the impact of current distribution and temperature are shown for magnetic recording heads. In magnetic recording anisotropic magnetoresistive (AMR) and spin valve devices are usually simulated with somewhat idealized shapes and current distributions. As recording density increases with its accompanying decrease in reader dimensions, modeling of the actual reader geometry with temperature effects becomes more important.

\section{MICROMAGNETIC ALGORITHM}

In this code structures of various shapes are defined on a nonuniform, Cartesian mesh. The magnetization is advanced using the Landau-Lifshitz-Gilbert dynamic equation [1]. Each structure is given material properties that are stored on the mesh nodes contained within the structure. The micromagnetic 
quantities include all three components of the magnetization, $M$, the saturation magnetization, $M_{\mathrm{s}}$, the exchange constant, $\mathrm{A}$, the crystalline anisotropy constant, $\mathrm{K}$, the anisotropy direction, $\hat{k}$, the linear interlayer coupling constant, $A_{e x}$, the biquadratic interlayer coupling constant, $B_{e x}$, and an anitiferromagnetic (AFM) pinning strength, $\mathbf{H}_{\mathrm{pin}}$. The effective field is given by

$$
H_{e f f}=\frac{2 A}{M_{s}^{2}} \nabla^{2} \mathbf{M}+\frac{2 K}{M_{s}^{2}}(\mathbf{M} \cdot \hat{k}) \hat{k}-A_{e x} \sum_{l} \hat{m}_{l}-2 B_{e x} \sum\left(\hat{m}_{l} \cdot \hat{m}\right) \hat{m}_{l}+\mathbf{H}_{\text {pin }}-\nabla \Phi_{M}+\mathbf{H}_{c u r r}
$$

Here $\Phi_{M}$ is magnetostatic potential, and $\mathbf{H}_{\text {cur }}$ is the field induced by currents.

Structures either have a magnetization, $\mathbf{M}$, or a linear permeability, $\mu$. The potential is calculated at the mesh nodes from the finite difference equivalent of the equation

$$
\nabla \cdot(\mu \nabla \Phi)=4 \pi \nabla \cdot \mathbf{M}
$$

On a Cartesian mesh iterative matrix solution techniques involving tridiagonal splittings of the matrix can still be used to solve this elliptic equation. With this formulation the efficient Dynamic Alternating Direction Implicit (DADI) method [1] is used to solve the matrix. The magnetostatic field, $\mathbf{H}_{\text {mag, }}$ is given by the gradient of the potential

$$
\mathbf{H}_{\text {mag }}=-\nabla \Phi
$$

\section{II.1 Currents and temperature}

The current induced field is found from the current density, the vector potential, and Ampere's law[1]. The current density, $\mathbf{J}$, can be defined as a constant in structures or calculated from the structure conductivities, $\sigma$. In the latter case the electrostatic potential, $\Phi_{\mathrm{e}}$, is found from

$$
\nabla \cdot\left(\sigma \nabla \Phi_{e}\right)=0
$$

The current density is given by Ohm's law

$$
\mathbf{J}=-\sigma \nabla \Psi_{e}
$$

The ends of the leads for conducting devices are given a Dirichlet boundary condition for the electrostatic potential. Insulating regions in the simulation are set at zero potential. At the interface between conducting and insulating matcrials a Neumann zero boundary condition is imposed on the potential 
causing current to be parallel to the interface. The total current for a device may be specified. In this case the code calculates the total current crossing a planar surface defined by the user. The potential across the device is then scaled to provide the correct current.

The resistivity, $\rho$, of AMR and giant magnetoresistive (GMR) structures is allowed to change depending on the local magnetization configuration. AMR depends on the angle, $\theta_{\mathrm{a}}$, between $\mathbf{J}$ and $\mathbf{M}$.

$$
\rho(\mathbf{x})=\rho_{o}+\Delta \rho \cos ^{2}\left(\theta_{a}(\mathbf{x})\right)
$$

GMR depends on the angle, $\theta_{g}$, between the pinned and free layers.

$$
\rho(\mathbf{x})=\rho_{o}+\Delta \rho_{o}\left[1-\cos \left(\theta_{g}(\mathbf{x})\right)\right] / 2
$$

The magnitude of $\Delta \rho / \rho$ is obtained from experimental results for particular AMR and GMR sheet films.

The temperature is solved using the heat equation and the previously calculated current density.

$$
\nabla(\kappa \nabla T)=-\rho \mathrm{J}^{2}
$$

The thermal conductivity is defined on the mesh according to the structure locations. The pinning of AFM pinned layers and the interlayer coupling for multilayered films are allowed to vary with temperature based on input files of experimental data. The local $\rho$ and $\Delta \rho$ are given a linear temperature dependence with the proportionality constant set by experimental data.

$$
\rho(\vec{x}, T)=\rho(\vec{x})(1+\alpha T)
$$

With the temperature dependence of the resistance there is a feedback in the heating [2]. The following algorithm is implemented for each applied magnetic field value. 1) Apply the external magnetic field and iterate the micromagnetic system to equilibrium. 2) Calculate the resistivities given the magnetization configuration and temperature. 3) Apply a voltage across the leads. 4) Calculate the potential. 5) Calculate the current densities. 6) Calculate the current across a defined surface. 7) Scale the voltage to get the desired current in the device. 8) Calculate the temperature using the current density. 9) If the change in the temperature or voltage is greater than a user defined error criteria, return to step (1). 11) Apply the next value of the external field.

\section{2 Enbedded curved boundary method in micromagnetics}

In this section we describe how the Embedded Curve Boundary (ECB) method [3] is implemented on the orthogonal, non-uniform mesh. With such a mesh object surfaces are usually constrained to the mesh 
nodes resulting in "stair step" boundaries. In constrast the ECB method allows the use of a structured mesh but includes smooth curved boundaries by changing the finite difference equations in prescribed ways. The application of the ECB method can improve simulation results without increasing computational requirements.

Specifically, second order finite differencing of the magnetostatic field equation at node $(i, j, k)$ (corresponding to $(x, y, z))$ gives:

$$
\begin{aligned}
& \frac{2}{\Delta x^{+}+\Delta x^{-}}\left[\frac{\mu^{+}}{\Delta x^{+}} \Phi^{+}-\left(\frac{\mu^{+}}{\Delta x^{+}}+\frac{\mu^{-}}{\Delta x^{-}}\right) \Phi^{0}+\frac{\mu^{-}}{\Delta x^{-}} \Phi^{-}\right]+\ldots \\
& =4 \pi\left[\frac{M_{x}^{+} \Delta x^{-}+M_{x}^{0}\left(\Delta x^{+}-\Delta x^{-}\right)-M_{x}^{-} \Delta x^{+}}{2 \Delta x^{-} \Delta x^{+}}+\ldots\right]
\end{aligned}
$$

where

$\Delta x^{ \pm}=|x(i)-x(i \pm 1)|$ is the discretization of the mesh.

$\Phi^{ \pm}=\Phi(i \pm 1, j, k) \Phi^{0}=\Phi(i, j, k)$ is the magnetostatic potential.

$M_{x}^{ \pm}=M_{x}(i \pm 1, j, k), M_{x}^{0}=M_{x}(i, j, k)$ is the $x$-component of $\mathbf{M}$.

$\mu^{ \pm}=.5(\mu(i, j, k)+\mu(i \pm 1, j, k)$ is the average linear permeability .

'...' refers to the differencing in the $y$ and $z$ directions.

Eq. (10) implicitly assumes there are no surfaces between node $(i, j, k)$ and any of its six closest neighbors. The ECB method adjusts the coefficients in the finite difference equation for a boundary located between the mesh nodes. Consider a simple surface between a magnetic structure with magnetic moment (Region 1) and empty space (Region 2) with $\mu=1$ as shown in Fig. 1. The basic boundary conditions for the magnetic fields, assuming no surface currents, can be manipulated to give:

$$
H_{x}^{(2)}-H_{x}^{(1)}=4 \pi \mathbf{M}_{\text {surface }} \cdot \mathbf{n} n_{x} \equiv \sigma_{\text {mag }}
$$

where the superscripts refer to the region. $H_{\mathrm{x}}$ is the $x$-component of the magnetostatic field. $\mathbf{M}_{\text {surface }}$ is the magnetization at the surface. The surface normal $\left(\mathrm{n}=n_{x} \mathrm{x}+n_{y} \mathrm{y}+n_{z} \mathrm{z}\right)$ is the unit surface normal from Region 1 to 2 at the point where the boundary intersects the line connecting node $(i, j, k)$ to node 
$(i+1, j, k)$. The magnetic potential at the surface, $\Phi^{*}$, is obtained by taking a first order difference of the potential on each side of the surface. The result from Eq. (11) is

$$
\Phi^{*}=\left[\delta\left(\Delta x^{+}-\delta\right) b_{\text {mag }}+\delta \Phi^{+}+\left(\Delta x^{+}-\delta\right) \Phi^{0}\right] / \Delta x^{+}
$$

where $\delta$ is the distance from the position $(i, j, k)$ to the position where the surface intersects the line connecting nodes $(i, j, k)$ and $(i+1, j, k)$. Eq. (10) can now be modified by differencing from the surface position instead of the node position, $(i+1, j, k)$. In other words $\Phi^{+}$is replaced with $\Phi^{*}, \Delta x^{+}$is replaced with $\delta$, and $M_{x}^{+}$is replaced with $M_{\mathrm{x} \text { surace }}$ (the $x$-component of $\mathbf{M}_{\text {surface }}$ ). The modification is completed by substituting Eq. (12) for $\Phi^{*}$. Rearranging terms gives

$$
\begin{aligned}
& \frac{2}{\delta+\Delta x^{-}}\left[\frac{1}{\Delta x^{+}} \Phi^{+}-\left(\frac{1}{\Delta x^{+}}+\frac{1}{\Delta x^{-}}\right) \Phi^{0}+\frac{1}{\Delta x^{-}} \Phi^{-}\right]+\ldots \\
& =4 \pi\left[\frac{M_{\text {x surface }} \Delta x^{-}+M_{x}^{0}\left(\delta-\Delta x^{-}\right)-M_{x}^{-} \delta}{2 \Delta x^{-} \delta}+\ldots\right]-\frac{2\left(\Delta x^{+}-\delta\right) b_{\text {mag }}}{\Delta x^{+}\left(\delta+\Delta x^{-}\right)}
\end{aligned}
$$

The $y$ and $z$ components remain unchanged. A similar equation can be derived for node $(i+1, j, k)$ in Region 2.

Constant potential or Dirichet boundary conditions may be imposed. For the case where region 2 is a structure of constant potential, $\Phi^{+}$is replaced with the constant potential, $\Phi_{D}$. Eq. (10) becomes:

$$
\begin{aligned}
& \frac{2}{\delta+\Delta x^{-}}\left[-\left(\frac{1}{\delta}+\frac{1}{\Delta x^{-}}\right) \Phi^{0}+\frac{1}{\Delta x^{-}} \Phi^{-}\right]+\ldots \\
& =4 \pi\left[\frac{M_{\mathrm{x} \text { surface }} \Delta x^{-}+M_{x}^{0}\left(\delta-\Delta x^{-}\right)-M_{x}^{-} \delta}{2 \Delta x^{-} \delta}+\ldots\right]-\frac{2 \Phi_{D}}{\delta\left(\delta+\Delta x^{-}\right)}
\end{aligned}
$$

Once again, the $y$ and $z$ components have not changed, and the right hand side has been changed to include the Dirichlet boundary value.

The preceding description is the general procedure for ECB to adjust the finite difference equations. Note that the derived coefficients continue to link only nearest neighbor mesh points in the finite difference template. Iterative matrix solution techniques involving tridiagonal splittings of the matrix can still be used to solve this elliptic equation. There remain three outstanding issues with this formulation. First, the derivation of Eq. (11), assumed no surface currents. As stated in the previous section, a separate algorithm 
[1] is used to find the current induced fields. The fields are then superimposed along with the other contributions to the effective magnetic field.

Second, and more important, the linear permeability of the non-magnetic structure was assumed to be unity. If $\mu$ is one or the surface normal lies along one of the coordinate axes, Eq. 4 is correct. In the later case the multiplication of $H_{x}^{(2)}$ by $\mu$ corrects Eq. (4). If the previous conditions are not met, Eq. 4 then has cross terms involving $H_{\mathrm{y}}, H_{\mathrm{z}}$, and $\mu$. While this is not fatal to the algorithm, it does mean that a simple tridiagonal matrix for each dimension is destroyed, and DADI cannot be employed to find $\Phi$. A later article will be published explaining the steps required to retrieve a tridiagonal system for arbitrary boundaries with permeabilities.

The third and final issue to be addressed is how to find the magnetic moment, $\mathbf{M}_{\text {surface }}$, at the surface since, in general, there is no node there. According to micromagnetic theory [4],

$$
\frac{\partial M}{\partial \mathbf{n}}=0
$$

Numerically, this constraint is imposed by projecting back along the surface normal into the structure a distance $2 \delta$ and linearly interpolating to find a value $\mathbf{M}_{\text {interpolatc }}$ as in Fig. 1. The linear interpolation is taken from the surrounding mesh points. The finite difference form of Eq. (15) becomes

$$
\frac{\mathbf{M}_{\text {surface }}-\mathbf{M}_{\text {inerpolate }}}{2 \delta}=0
$$

$\mathbf{M}_{\text {surfacc }}$ is simply the interpolated value. The finite difference equations have now been altered to allow for curved boundaries. $\mathbf{M}_{\text {surface }}$ is also used in the exchange, $\nabla^{2} \mathbf{M}$, term of Eq. (1). Each node can have up to six surfaces surrounding it. The coefficients are changed according to the above prescription depending on whether nodes arc located inside or adjacent to structures.

\section{SUBMICRON-SCALE CO DOTS}

Micromagnetic simulations were used to simulate the magnetic response of polycrystalline Co elliptical dots of varying thickness (160-400 $\stackrel{\circ}{)}$ ). The dots have major and minor axes of $450 \mathrm{~nm}$ and $250 \mathrm{~nm}$, respectively. The crystalline anisotropy is assumed to be random in the $x y$ plane with coefficient 
$K=7.5 \times 10^{5} \mathrm{erg} / \mathrm{cc}$. The grain size is approximately $60 \stackrel{\circ}{\AA}$, and $A=1.6 \times 10^{-6} \mathrm{erg} / \mathrm{cm}^{2}$. Detailed experimental procedures and results can be found in [5].

Fig. 2 shows the calculated and measured coercivity of the dots in the easy direction for different thickness. The magnetic moment is not known precisely, but measurement indicates an effective moment with a lower bound of $1160 \mathrm{emu} / \mathrm{cc}$ which is less than the bulk value of $1450 \mathrm{emu} / \mathrm{cc}$. Simulations were done with $M_{\mathrm{s}}$ of 1450 and $1160 \mathrm{emu} / \mathrm{cc}$. Simulations using ECB were compared to simulations using stair stepped boundaries on the same computational mesh. Several simulation points are shown for each thickness. The different points result from different random distributions for the grain anisotropy directions. ECB and stair stepped boundaries give similar coercivity results for each value of $\mathrm{M}_{\mathrm{s}}$. The simulations with the lower $\mathrm{M}_{\mathrm{s}}$ are in closer agreement with experiment.

Differences are more evident in the magnetization configurations. Experimental results suggest a single vortex state exists during switching $97 \%$ of the time for all thickness'. This state is evident in MFM images of the dots[5]. In the simulations different magnetization configurations occurred for different initializations of the grain anisotropy distribution. The stair step simulations produced a single vortex state only for the thinnest structures. All other thickness exhibited very complex magnetization patterns. On the other hand, the ECB method produced single vortex states at all thickness'. A few double vortex states occurred at the intermediate thickness. In Fig. 3, the hysteresis loops from experiment for $160 \mathrm{~A}$ (thick line) and $400 \mathrm{~A}$ (thin line) dot are shown. The ECB simulations results are given as squares for the $160 \mathrm{~A}$ dots and triangles for the $400 \mathrm{~A}$ dots. The simulation curves are averaged over all simulations that exhibited a single vortex. The ECB method provides a very close fit to experiment for the coercivity and denucleation. Some error is evident in the nucleation field of the vortex. Nucleation has been seen to be dependent on the coupling between grains which is not known precisely. As a whole these results indicate that the ECB method can simulate particles of various shapes with good accuracy.

\section{GMR Read Heads}

This section presents the results of an example read head simulation to demonstrate the ability of the code to include the combined effects of current and temperature on a read head. A dual spin valve [6] was modeled to minimize the effect of the current induced field on the magnetic response of the head. The GMR layers are /AFM 50A/CoFe 20A/Cu 25A/free layer 50A/Cu 25A/CoFe 20A/AFM 50A/. The frec 
layer Mst is $0.5 \mathrm{memu} / \mathrm{cm}^{2}$. The physical track width is $0.5 \mu \mathrm{m}$ with a stripe height of $0.4 \mu \mathrm{m}$. device was given a sheet resistance of $15 \Omega / \mathrm{sq}$ and a GMR response of $10 \%$. The shield to shield gap is $0.13 \mu \mathrm{m}$.

The antiferromagnetic (AFM) pinning layers were given a temperature dependence similar to the IrMn in Fuke, et al [7]. The AFM pinning is $600 \mathrm{Oe}$ at room temperature and is zero at the blocking temperature of $260^{\circ} \mathrm{C}$. The response of the device is shown for a media having an Mrt of $0.5 \mathrm{memu} / \mathrm{cm}^{2}$ and a total magnetic spacing of $500 \mathrm{~A}$. For this device the temperature coefficient, $\alpha_{1}$, for $\rho$ was set to $0.1 \% /^{\circ} \mathrm{C}$, and the temperature coefficient, $\alpha_{2}$, for $\Delta \rho$ was set to $-0.2 \% /{ }^{\circ} \mathrm{C}$. The thermal conductivity of insulating materials was $1.0 \mathrm{~W} /{ }^{\circ} \mathrm{C}-\mathrm{m}$, and the thermal conductivity of the other materials was $35 \mathrm{~W} /{ }^{\circ} \mathrm{C}-\mathrm{m}$.

In the simulations the ambient temperature was set at $50^{\circ} \mathrm{C}$ as an approximation to the elevated temperature in a hard drive. Figure 4 is a contour plot of the temperature in the free layer for $6 \mathrm{~mA}$ of sense current. Superimposed on the contours are the magnetization vectors. Note that the pinned layers of the dual spin valve tend to bias the free layer magnetization downward. As expected the temperature is a maximum at the center of the stripe $\left(100^{\circ} \mathrm{C}\right)$ and a minimum at the junction with the leads $\left(67^{\circ} \mathrm{C}\right)$. The temperature falls off at the top of the stripe as heat conducts through the gap materials. The temperature remains uniform at the insulating air bearing surface (ABS).

As the current in increased the temperature of the GMR stripe increases at nearly a quadratic rate [8]. The average temperature of the device is shown in Fig. 5. This increase in temperature affects both $\Delta \rho$ of the GMR and the pinning of the AFM layer. Fig. 6 shows the peak-to-peak response of the device as the current is increased. Curve a) is the response without temperature effects. It is almost linear with current. Curve b) simulations included the change in $\Delta \rho$ with temperature. Curve c) is the product of curve a) and $\left(1+\alpha_{2} T(I)\right)$ where $T(I)$ is taken from Fig. 5. The combined simulation, curve b), has a response that is slightly better than the approximation of curve $c$ ). With the inclusion of pinning temperature dependence the response drops further as shown in curve d). The response of the case d) is \% lower than the response where heating is neglected.

\section{Conclusion}

The combination of smooth surfaces, current distributions, and heating effects in a micromagnetics algorithm provides the capability to model magnetic particles, read heads, etc. with realistic shapes and 
under actual operating conditions. The algorithm is formulated in a way that allows the use of the finite difference method and efficient matrix solution techniques. The additional capability of this micromagnetic algorithm will lead to helpful insights on the operation of high density, recording heads.

\section{ACKNOWLEDGEMENTS}

Part of this work was performed under the auspices of the United States Department of Energy by the Lawrence Livermore National Laboratory under Contract W-7405-ENG-48.

\section{RFFERENCES}

[1] M.R. Gibbons, J. Magn. Magn. Mater., 186 (1998) 389.

[2] K.F. Young, IBM J. Res. Develop., 34 (1990) 706.

[3] D.W. Hewett, J. Comput. Phys., 138 (1997) 585.

[4] W.F. Brown, Micromagnetics, Wiley, New York, 1963.

[5] A. Fernandez, M.R. Gibbons, M.A. Wall, and C.J. Cerjan, J. Magn. Magn. Mater.,

[6] T.C. Anthony, J.A. Brug, S. Zhang, IEEE Trans. Magn., 30 (1994) 3819.

[7] H.N. Fuke, K. Saito, Y. Kamiguchi, H. Iwasaki, and M. Sahashi, J. Appl. Phys., 81 (1997) 4004.

[8] Y. Guo and K. Ju, IEEE Trans. Magn., 33 (1997) 2917.

\section{FIGURES}

1. Geometric definitions for the embedded curved boundary (ECB) method at surfaces.

2. Easy axis coercivity for dots of various thickness.

3. Magnetization in the easy direction for simulation (symbols) and experiment (lines).

4. Temperature contours in the free layer and magnetization configuration at $6 \mathrm{~mA}$. The temperature is $67^{\circ} \mathrm{C}$ at the edges and $100^{\circ} \mathrm{C}$ in the center.

5. Temperaturc as a function of current in the dual spin valve.

6. Response of the dual spin valvc as a function of current: a) no temperature effects, b) $\Delta \rho$ affected by temperature, c) curve a) multiplied by $\left(1+\alpha_{2} T(I)\right)$, and d) both $\Delta \rho$ and $\mathrm{H}_{\mathrm{pin}}$ affected by temperature. 


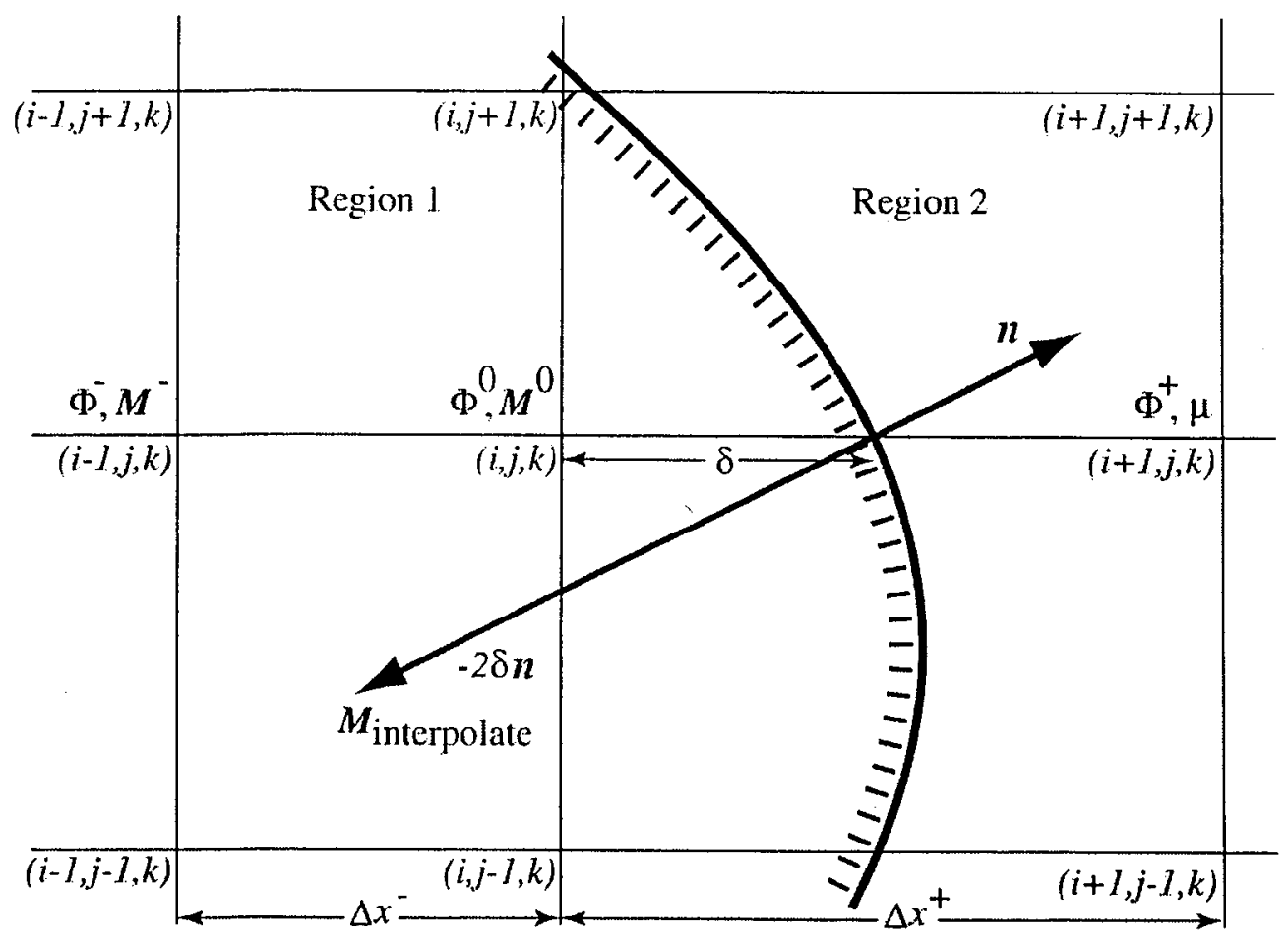

Figure 1: Geometric definitions for the embedded curved boundary (ECB) method at surfaces.

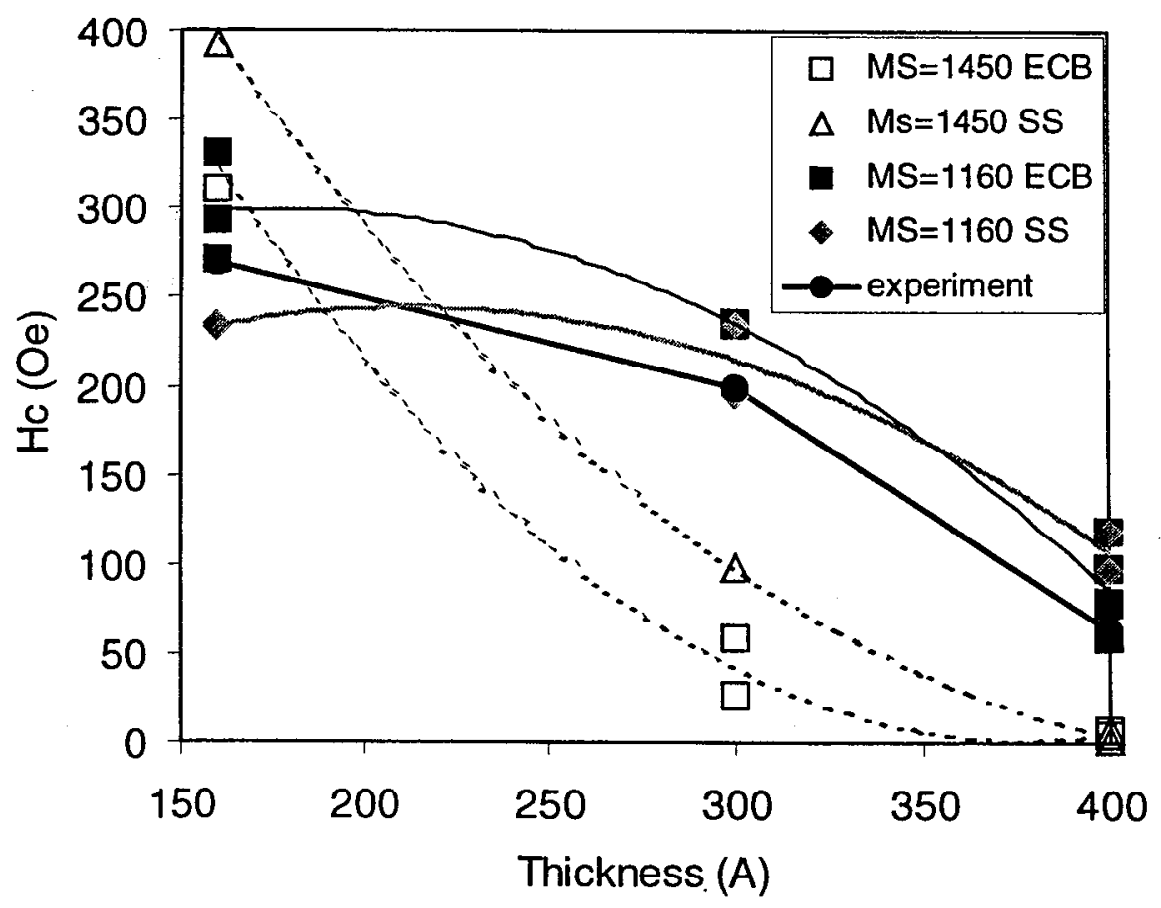

Figure 2: Easy axis coercivity for dots of various thickness. 


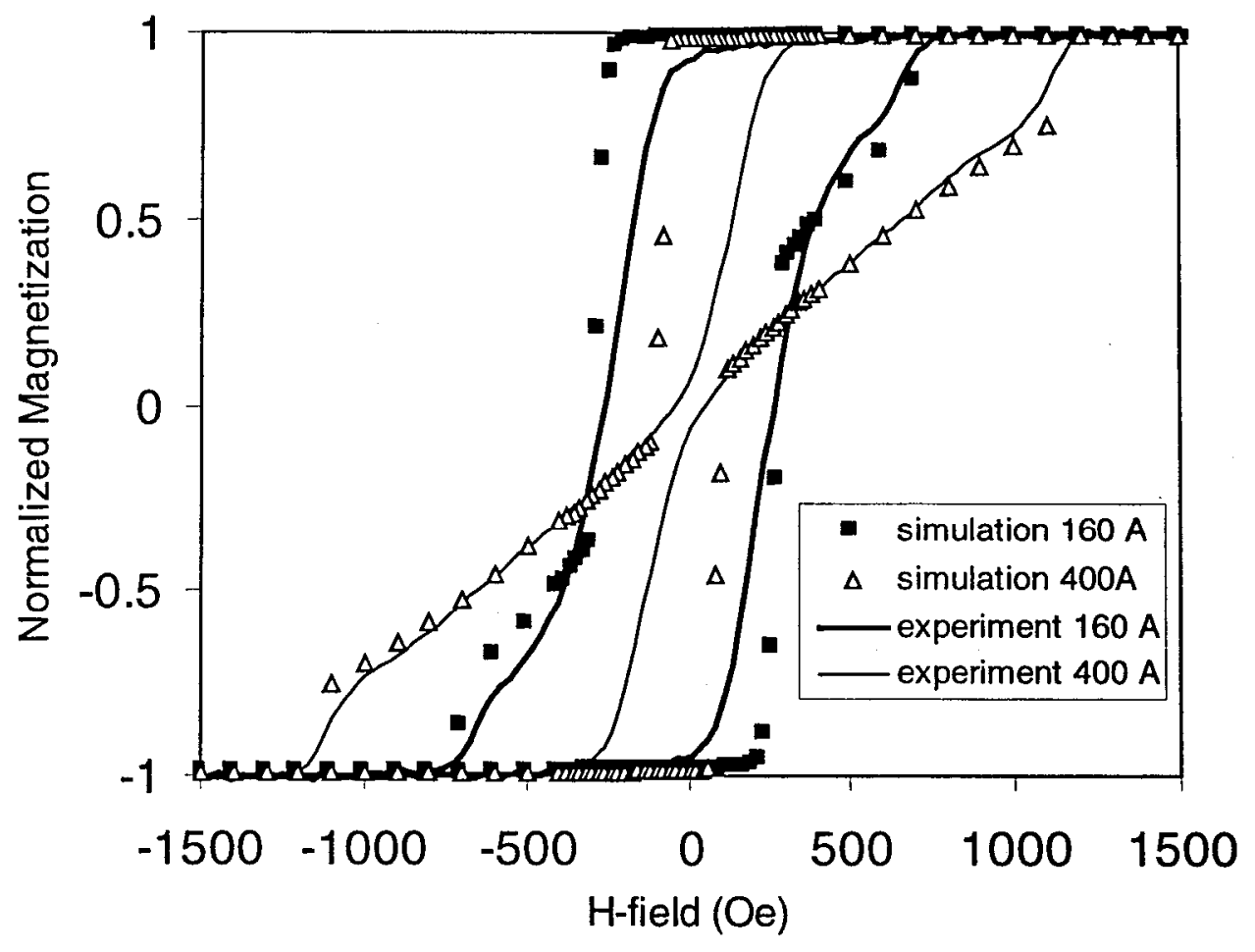

Figure 3: Magnetization in the easy direction for simulation (symbols) and experiment (lines).

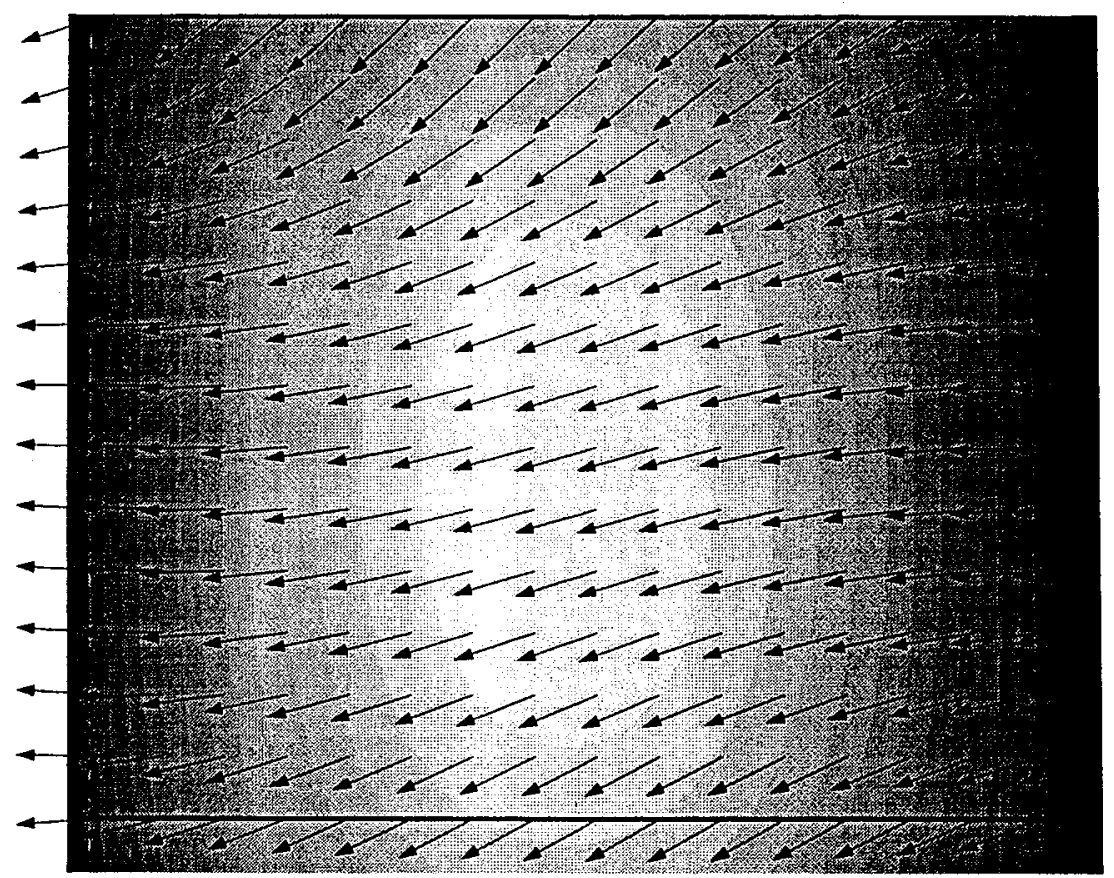

Figure 4: Temperature contours in the free layer and magnetization configuration at $6 \mathrm{~mA}$. The temperature is $67^{\circ} \mathrm{C}$ at the edges and $100^{\circ} \mathrm{C}$ in the center. 


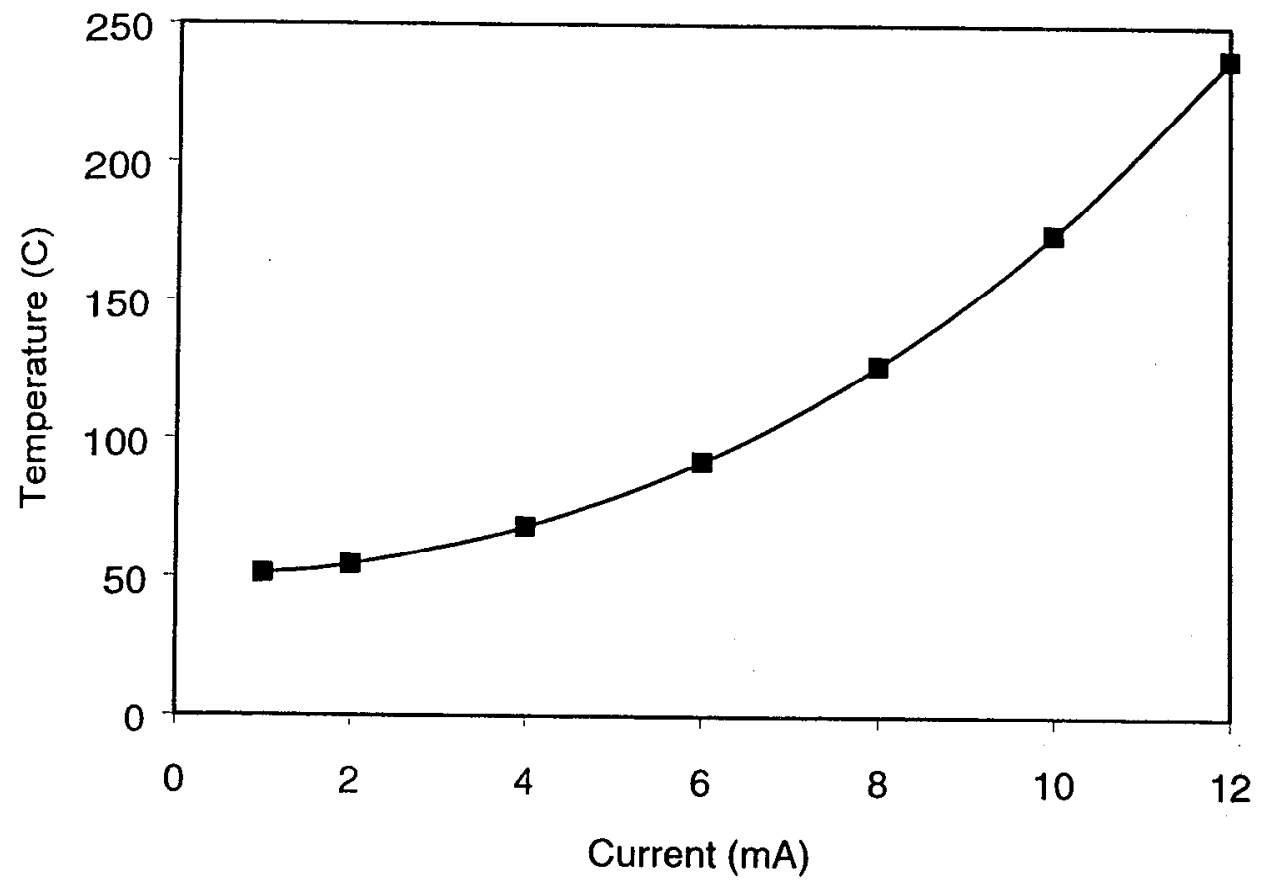

Figure 5: Temperature as a function of current in the dual spin valve.

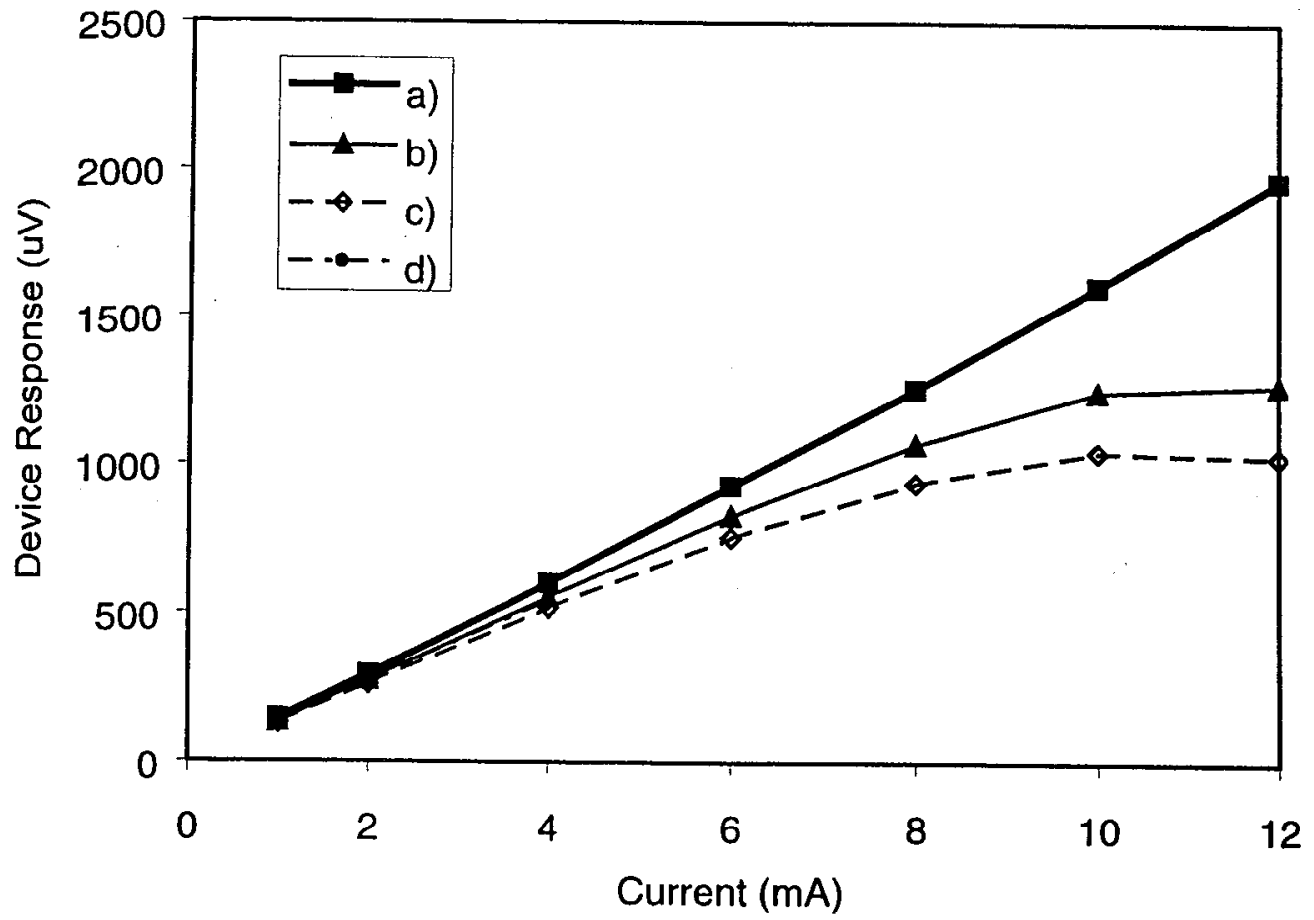

Figure 6: Response of the dual spin valve as a function of current: a) no temperature effects, b) $\Delta \rho$ affected by temperature, c) curve a) multiplied by $\left(1+\alpha_{2} T(I)\right)$, and d) both $\Delta \rho$ and $\mathrm{H}_{\text {pin }}$ affected by temperature. 